\title{
Eva Mameli y Mario Calvino: una presencia soslayada en el México revolucionario
}

\author{
M. Cristina Secci \\ Universidad de Cagliari \\ mcristinasecci@yahoo.it
}

\begin{abstract}
Resumen: Según el testimonio de Eva Mameli, esposa y colega del científico agrónomo Mario Calvino, padres del escritor Italo Calvino, fue el embajador mexicano Joaquín Casasús quien en un viaje a Italia conoció los trabajos del entonces joven catedrático Calvino, y le propuso trabajar en México, consiguiéndole el nombramiento de jefe de la División de Horticultura de la Estación Agraria Central. Gracias en parte a la consulta del recientemente restaurado en Cuba "Expediente Sra. Eva Mameli de Calvino", es posible documentar la estancia en América Latina del matrimonio Calvino, pioneros en estudios de botánica y agricultura en México desde 1909 y en Cuba desde 1917.
\end{abstract}

Aвstract: According to Eva Mameli's testimony, the wife and colleague of the scientist and agronomer Mario Calvino, Italo Calvino's parents, it was the mexican Embassador Joaquín Casasús who during a trip to Italy became acquainted with the then young italian profesor Calvino, and invited him to work in México, as Jefe de la División de Horticultura de la Estación Agraria Central. The recently restored "Expediente Sra. Eva Mameli de Calvino" in Cuba, gives the autor the possibility to document the years spent in Latinoamerica of the Calvino matrimony, pioneers in botanical and agricultural studies in México since 1909 and in Cuba since 1917.

Palabras Clave: biografía; Mameli; México; revolución; Cuba; Calvino.

KeYwords: Biography; Mameli; México; Revolution; Cuba; Calvino.

FECHA DE RECEPCIÓN: 4 de febrero de 2015

FECHA DE ACEPTACIÓN: 17 de marzo de 2015

Nací cuando mis padres estaban a punto de regresar a Italia después de haber pasado varios años en el Caribe; de ahí la inestabilidad geográfica que constantemente me obliga a desear ir a otros lugares.

Italo Calvino, Ermitaño en París

$S_{\text {diferencia de que se establecen por cinco años al otro lado del océa- }}^{\text {e casan y se van a Cuba, como en una clásica luna de miel. Con la }}$ 
no, en la Estación Experimental Agronómica de Santiago de las Vegas, y cuando regresan llevan consigo un valioso acervo de información agro-botánica que utilizarán en San Remo, además de dos hijos que darán mucho de qué hablar: Italo, el escritor, y Floriano, el geólogo. ${ }^{1}$ De los dos, Italo fue quien marcó la propia distancia respecto a la cultura verde y científica con la cual creció y se formó: "En mi familia solo se les rendían honores a los estudios científicos. Tuve dos tíos químicos; mi hermano es geólogo. Yo soy la oveja negra, el único literato de la familia" (Calvino 1994: xi). O tal vez fue quien en la familia se portó de manera más vegetal, nutriéndose de sus raíces, transformándolas, por lo cual, la reconstrucción de la vida de sus padres puede resultar de agradable utilidad tanto para los estudiosos de su obra como para los apasionados de la historia de la ciencia.

No hay que olvidar que Italo Calvino, como colaborador de la editorial Einaudi, dio gran impulso a la difusión en Italia de la narrativa latinoamericana, y que su contribución se debe, además de la retroalimentación consecuente a sus lecturas y viajes, a cierta vivencia familiar y al aire centroamericano respirado en su niñez. Son palabras de Italo:

Mi madre era una mujer muy estricta, austera y rígida en sus ideas, tanto en las pequeñas como en las grandes cosas. También mi padre era muy austero y huraño pero su severidad era más ruidosa, colérica, intermitente. Mi padre, como personaje de ficción es más fácil de lograr, bien como viejo ligur muy arraigado a su tierra, bien como hombre que había viajado por el mundo y que había vivido la revolución en tiempos de Pancho Villa. Eran dos personalidades muy fuertes y bien definidas (x).

¿Quiénes eran esos padres que podían al mismo tiempo pertenecer al universo de la ficción narrativa y a la ciencia?

Se trata de Eva Mameli y Mario Calvino, quienes se casaron por lo civil en Pavía el 30 de octubre de 1920 (la participación de boda anuncia el 1 de noviembre $)^{2}$ y, después de una breve estancia en San Remo anunciada en el periódico local, ${ }^{3}$ se fueron a La Habana.

\footnotetext{
${ }^{1}$ Floriano Calvino dirigirá el Instituto de Geología de la Universidad de Génova, y será uno de los expertos consultados en ocasión del desastre del Vajont.

${ }^{2}$ Un agradecimiento a Loretta Marchi, directora de la Biblioteca de San Remo, que me enseñó el documento.

3 "Hoy, sábado, nuestro óptimo amigo el Prof. Mario Calvino se casó en Pavía con
} 
Del encuentro de la pareja se cuenta una historia más bien extravagante, una epopeya que tiene todo el sabor de una fantasía romántica. El manuscrito inédito de Domenico Aicardi ${ }^{4}$ (cordialmente concedido a mí por Gerson Maceri) $)^{5}$ proporcionó los particulares de aquel encuentro.

Según la versión de Aicardi, Mario Calvino viajó en 1920 de Cuba —donde vivía y trabajaba desde 1917- a Italia para una breve misión. En esa ocasión se dirigió al Ministerio de Agricultura y solicitó información sobre una botánica soltera para casarse y llevarla al otro lado del océano. Dice Aicardi: "Calvino aprovechó ese viaje a Italia para casarse". Entre los nombres sugeridos estaba el de Eva Mameli, entonces docente en la Universidad de Pavía y, según la anécdota, fue el único tomado en consideración. Mario tomó un tren directo hacia Pavía para tocar en la puerta de la casa de Eva y, sin demora, pedirle que se casara con él delante de los ojos atónitos de la madre. Eva, también asombrada por el "modo tan poco ortodoxo" con el que se pedía su mano, solicita tiempo para poder tomar una decisión. Pero la prisa de Calvino impuso una rápida respuesta: "El entendimiento fue fácil y el matrimonio, solo por lo civil, rápido, teniendo ambos las mismas ideas en cuanto a la religión", escribe Aicardi.

Este es el mito sobre la pareja, referido también por Jean Henri Nicolas en un libro de $1937,{ }^{6}$ aunque otra versión sugerida por Paolo Monelli en un artículo dedicado a Mario (citado en Valle 1969)

la doctora Eva Mameli, docente de botánica en aquella universidad. La doctora Mameli, que es una amable y bella señorita nacida en Cerdeña, ya ha publicado numerosos y apreciadísimos trabajos y ha relacionado su nombre con importantes descubrimientos de fisiología y microscopía vegetal. La pareja verdaderamente privilegiada, después de transcurrir pocos días en San Remo, partirá hacia La Habana (Cuba), donde el Prof. Calvino reanudará su alto ministerio, del que se ausentó tras pedir licencia" (Eco de la Riviera, 31 de octubre de 1920). Citado por Marchi 2012.

${ }^{4}$ Domenico Aicardi, Mario Calvino.

${ }^{5}$ Gerson Maceri, Mario Calvino, biografia di un progressista utopico.

6 "I was told the romantic manner in which Dr Calvino married his wife. Appointed to Mexico, he was ready to go; the steamer was in Genoa making ready to sail. Dr Calvino noticed that his transportation was for him 'and wife', but he was a bachelor. He inquired where in Italy was the best woman professor of botany. He was told at the Milan University. He hurried there, called at the lady's home and introduced himself thus: 'I am Dr Calvino. I am sent to Mexico. I have come to marry you'. The young woman, startled, answered, 'But, Doctor Calvino, I do not know you. I must have a week to think over your proposal'. 'Madame, I cannot wait a week; the ship is sailing. 
confirma el viaje para una misión agro-matrimonial y menciona a una jovencita originaria de la isla de Cerdeña con licenciatura en ciencias naturales cortejada por el agrónomo antes de emigrar, tal vez durante sus años de la universidad. Posteriormente, el conocimiento podría haberse profundizado gracias a una correspondencia entre ambos sobre temas científicos en común (Marchi: 243). En suma, las ciencias fueron cómplices de su encuentro.

¿Pero quiénes son y de dónde provienen los protagonistas de esta historia? Mario Calvino había nacido en San Remo el 26 de marzo de 1875, mostrando muy pronto un carácter inquieto: si en el liceo su promedio no llegaba al siete, y su nota de conducta dejaba mucho que desear, sería en Pisa, con una licenciatura con mención honorífica, donde comenzará a revelar su propia inclinación hacia las ciencias, que lo llevará a ser un eminente agrónomo. La misma Eva lo retrata de forma apasionada:

La vida del muchacho fue libre, más bien salvaje: las tendencias del adolescente rápidamente pasaron, de las bellezas naturales que lo rodeaban y de la pasión de su padre por la agricultura y los estudios humanísticos, al amor por el campo, a la observación de las plantas y de los animales, al estudio. [...] Los cultivos florales se encontraban en sus inicios y el neoagrónomo estaba poseído por una fiebre de iniciativas, no solo en el campo florícola, sino al mismo tiempo en el olivícola, vitícola $y$, con el ardor de los generosos, también en el campo social (Mameli Calvino: I).

Explorador pragmático, Mario era también un hombre influyente cuya herencia masónica y actividad agrónoma lo llevó a tejer una fina red de relaciones en todo el mundo con gobernadores, industriales, políticos y dictadores. Un hombre descrito como ecléctico e inconforme, que de su profesión, como bien dijo Eva en su sincera esquela mortuoria, "hizo un apostolado para el que asumió como propio el lema de Eliseo Reclus: 'conocer la verdad y regarla a los cuatro vientos'”. Y los cuatro vientos fueron también los cuatro puntos cardinales: en 1909 Mario se trasladó a México y en 1917 a Cuba, con diversos viajesmisión intercalados a California, Texas, Florida y luego Brasil, Estados

We must be married at once', he said. 'All right', she replied, 'I will do it'. They were married and, as fairy tales always end, 'they lived happily ever after'” (Nicolas: 54-55). 
Unidos, Hawai, Rodi, Somalia. Según narra Eva, durante un viaje a Italia el embajador mexicano Joaquín Casasús quedó tan sorprendido por el entusiasmo y lo práctico de las propuestas del joven catedrático, que le pidió que repitiera tales conceptos en México y le ofreció el nombramiento de jefe de la División de Horticultura de la Estación Agrícola Central. En una nota publicada en el periódico La Agricultura en Liguria, el propio Calvino informa a sus lectores sobre su inminente aventura en México: "Un pueblo de fuertes me ha extendido los brazos fraternos y me ha invitado a trabajar para él en extensas tierras vírgenes y muy fértiles" (31 de diciembre de 1908).

Otro interesante ensayo de Stefano Adami (2010), quien cita un fascículo resguardado en el Archivo Central del Estado de Roma, parece sugerir otras perspectivas al reconstruir el llamado affaire Calvino: el 21 de febrero de 1908, un año antes de su llegada a México, el Corriere della Sera publicó un artículo que decía que el frustrado atentado contra el zar Nicolás II era obra de un italiano, un cierto Mario Calvino. Pero, como se revelaría en los días sucesivos, se trataba más bien del revolucionario ruso Lebedintsev, exiliado en Italia entre 1905 y 1907, y arrestado cuando reingresó a San Petersburgo en posesión de un pasaporte italiano a nombre de Mario Calvino. Lebedintsev terminaría sus días con una condena a la horca junto con sus compañeros. Para Mario el suceso representó un acontecimiento incómodo, nunca del todo aclarado, pero probablemente será una de las razones que lo empujarán a cambiar de aires y partir rumbo a México, como revelará posteriormente el mismo Italo:

El "caso Calvino" renovó la hostilidad hacia mi padre en los ambientes conservadores y clericales locales (él era un personaje muy característico de la época: apóstol de la educación agrícola, fundador de haciendas cooperativas, director de la revista L'Agricoltura Ligure, obstinado anticlerical). La vida en Porto Maurizio se hizo difícil para él y en 1909 partió hacia México, donde le habían ofrecido la dirección de la Estación de Agronomía nacional (Calvino 2000: 1379).

En México orienta sus actividades hacia la experimentación de nuevos cultivos y a divulgar los conocimientos ya adquiridos: fundamental resulta su labor para que nazcan campos agrícolas experimentales en varios territorios y estados de la República. Después de solo tres años 
de permanencia, Calvino publicó un importante proyecto didáctico dirigido a los agricultores mexicanos que sintetizaba las necesidades de ese entonces: a los efectos de un progreso era preciso organizar una enseñanza agrícola ambulante, como se había hecho en Italia. Mario recorrió el país a lo largo y ancho en dos ocasiones por lo que "ninguno de los numerosos problemas de la agricultura de México, tierra de muchos climas, le fue ignoto" (Mameli Calvino: IV).

En su largo viaje por la agricultura y tierra mexicana, Calvino colabora en la columna "Sección de Agricultura" del Diario de México, con la revista especializada Hacienda y Ranchos y con el semanal Boletín de la Sociedad Agraria Mexicana, de la que fue también secretario; publica además una serie de circulares y boletines de la Estación Agrícola Central, así como volúmenes monográficos, algunos de los cuales conservan cierta utilidad.

En 1915 fue nombrado jefe del Departamento de Agricultura del Estado de Yucatán y encargado de la enseñanza agrícola ambulante en las poblaciones mayas. El 5 de diciembre de 1915 da el discurso oficial para la inauguración de la Escuela Agraria en Mérida:

Es para mí una verdadera satisfacción poder tomar parte en la inauguración de la Escuela de Agricultura de Yucatán, no sólo en mi calidad de Jefe del Departamento de Estudios Agrícolas de la Secretaría de Fomento, Colonización e Industria del gobierno central revolucionario, sino también como ciudadano del mundo y hombre que ha dedicado, por afición y convencimiento, toda su vida a las ciencias agrícolas y a su vulgarización a través de los continentes. $Y$ esto depende del hecho de que yo veo todo el alcance revolucionario de la generalización de los estudios agrícolas, porque ellos vuelven a las masas, desviadas y encorraladas en ciudades por los intereses de los monopolios, a la visión exacta del derecho humano sobre la tierra (Calvino 1915).

Y debido al temperamento que tenía, dice Eva, acompañó los proyectos de progreso agrícola con ideas de alto patriotismo y de audacia sociológica: "Una hamaca fue todo su equipaje en aquel borrascoso período, y un intérprete su compañero de viaje, ya que entre los campesinos mayas no era familiar la lengua española" (Mameli Calvino: $\mathrm{V}$ ).

En 1916 México estaba sacudido por el movimiento revolucionario: el 9 de marzo de ese año Pancho Villa conducía su conocida expedición contra la ciudad de Columbus, Nuevo México, provocando la 
reacción armada estadunidense. Mario Calvino, "con una muy noble carta para el Gobernador del Estado de Yucatán, ofreció su brazo como simple soldado por la causa del pueblo mexicano oprimido" (V). Pero en 1917 "las revoluciones sucesivas hicieron imposible los estudios", y después de un breve regreso a Italia, Mario se trasladó a Cuba, un país próspero "no obstante el casi absoluto monocultivo de cańa de azúcar, y sobre todo, de tabaco y café; esta prosperidad se debía a los altos precios del azúcar a causa de la guerra en Europa" (V). He aquí la descripción autobiográfica narrada por Calvino a Aicardi: "En un viaje a Cuba había visitado aquella tierra rica y próspera, y habiéndome hecho una propuesta muy favorable, la oferta de la dirección de las escuelas de los oficiales agrícolas de la Cuba Cane Sugar Corporation, un buen día decidí, después de casi diez años de permanencia en México, trasladarme a Mercedes, Santiago de las Vegas" (Aicardi: 6). Muy rápido le fue confiada la dirección de la Estación Experimental de Agronomía de Santiago de las Vegas para una reestructuración de la misma; de hecho, el desempeño de Calvino renovó su prestigio internacional.

Cuando Mario se casa con Eva Mameli, ella ya era una brillante mujer de ciencia, con una sólida preparación científica y una total dedicación al estudio y a la investigación. Había nacido en Sassari, Cerdeña, el 12 de febrero de 1886, en el seno de una familia laica y republicana, descendiente de Goffredo Mameli, autor del himno nacional italiano. Nicola Valle, sobrino de Evelina, como la llamaban en familia, la define en un artículo publicado en el 1969 como "perteneciente a una antigua familia, descendiente de Goffredo y bisnieta del arqueólogo Giovanni Spano". 7

En Cerdeña la joven Eva fue de las primeras muchachas en asistir a un liceo público, normalmente reservado a los hombres. En su época eran, de hecho, poquísimas las niñas que podían seguir sus estudios más allá de los nueve años previstos de escuela obligatoria. Esa misma tenacidad y anticonformismo la llevarán a convertirse en una de las más grandes científicas italianas del siglo xx. De hecho, fue una de las primeras mujeres en Italia en obtener la licenciatura en Ciencias Naturales y obtener la habilitación para la docencia en 1915. Aunque se haya atribuido esa primacía más veces a la botánica sarda Rina Monti, la pri-

${ }^{7}$ Giovanni Spano (1803-1878) es un ilustre arqueólogo y lingüista de Ploaghe, lugar de nacimiento de la madre de Eva. 
mer mujer en obtenerla en Italia. ${ }^{8}$ Es la misma Mameli desde Cuba, en un artículo publicado en la Revista de Agricultura, Comercio y Trabajo, quien menciona a diversas mujeres exponentes de la Universidad de Pavía que enaltecen las publicaciones y las metas académicas. ${ }^{9}$

Después de la muerte de su padre, Eva dejó Cerdeña para trasladarse junto a su madre Maddalena Cubeddu a Pavía, donde su hermano Efisio $^{10}$ era docente de química orgánica y en donde se inscribirá en Ciencias Naturales. En Pavía destacaban la cultura y la ciencia: Lazzaro Spallanzani había fundado allí en 1771 un museo de ciencias naturales famoso en todo el mundo por las colecciones de animales exóticos y, además, en Pavía vivía Camillo Golgi, el primer italiano en recibir en 1906, algunas semanas antes de Carducci, el Nobel de Medicina y Fisiología por sus estudios de histología del sistema nervioso y de la célula.

Siempre en Pavía, y durante su estancia en la universidad, Eva tuvo la oportunidad de frecuentar el prestigioso laboratorio criptogámico de Giovanni Briosi dedicado al estudio poco difundido en Italia de algas y musgos, considerados fundamentales en los estudios de fisiología, patología y ecología vegetal. Fue asistente voluntaria en el Instituto de Botánica y obtuvo el adiestramiento para la enseñanza sin nunca abandonar la investigación. Durante aquellos años profundizó junto a su hermano Efisio y su cuñada Anna Menessier en los aspectos químico-farmacéuticos de las plantas medicinales sardas. Sus artículos aparecieron en varias revistas académicas internacionales y su notoriedad aumentó, sobre todo, por un estudio publicado junto a Guido Pollacci en 1909 —año de la partida de Mario a México- sobre la fijación del nitrógeno atmosférico a través de órganos provistos de clorofila en especies no leguminosas (Mameli y Pollacci 1908 y 1911).

Durante la Primera Guerra Mundial Eva disminuyó el ritmo de la investigación para obtener el diploma de enfermera en la Cruz Roja italiana. Mientras su hermano Romualdo era prisionero de los austria-

${ }^{8}$ En 1899 , en anatomía y fisiología comparada.

9 "Rina Monti estudió Ciencias Naturales en Pavía, en donde se graduó de doctora en 1892; obtuvo en 1899 el título de Profesora Agregada y continuó en sus progresos académicos, hasta salir vencedora en la oposición para ocupar la cátedra de Zoología” (Mameli 1921a: 602).

${ }^{10}$ Efisio estuvo también entre los fundadores del independentista Partido Sardo de Acción. 
cos y Efisio se ocupaba del laboratorio químico de la Tercera Armada (Marchi: 242), Eva prestó servicio como enfermera voluntaria en la unidad de enfermos de tifo del hospital de reserva Ghislieri de Pavía. Su dedicación al cuidado de los enfermos fue reconocida con una medalla de plata de la Cruz Roja y con una de bronce del Ministerio del Interior a los beneméritos de la salud pública. En 1919 obtuvo otro prestigioso reconocimiento: el premio para las ciencias naturales de la Accademia Nazionale dei Lincei, institución que premiará posteriormente la narrativa de su hijo.

Mameli escribió y publicó más de 200 artículos científicos durante su carrera, además de un diccionario etimológico de los nombres genéricos y específicos de plantas y flores, y fundó junto con Mario las revistas Il giardino fiorito (1931-44 y 1946-47) y La Costa Azzurra agricola e floreale (1925-43). De su intervención recordamos también la reforestación con palmas, eucaliptos, encinos y otras plantas exóticas del jardín botánico de Cagliari que había sido gravemente dañado por la guerra; y los estudios de botánica aplicada (en particular los del tabaco y la caña de azúcar) durante los años que pasó en Cuba, primero en la Estación Experimental de Santiago de las Vegas y después en la Estación de Chaparra donde, como jefa del departamento de botánica en ambas estaciones, se convirtió en la primera mujer en la isla caribeña en ocupar un cargo directivo en el campo de la agricultura (Díaz Marreno: 89-116). Están muy bien detalladas las relaciones, depositadas y cuidadas en los archivos de INFIAT, ${ }^{11}$ en las que Eva periódicamente presentaba algún registro de las actividades desarrolladas por el propio departamento - hoy conservados en volúmenes ordenados por año-, así como de las visitas recibidas, de las adquisiciones y de los datos botánicos recogidos en la Estación (Secci 2014).

Recientemente restaurado, el Expediente Sra. Eva Mameli de Calvino (también custodiado en el archivo del INFIAT) nos confirma el calendario de sus principales actividades durante los años pasados en Cuba. Allí encontramos de nuevo las misiones autorizadas y las licencias recibidas, y el nombramiento por decreto ministerial del primer cargo de dirección del departamento de botánica del 1 de diciembre de 1920 “en

\footnotetext{
${ }^{11}$ Instituto de Investigaciones Fundamentales en Agricultura Tropical, nombre actual de la antigua Estación Experimental Agronómica de Santiago de Las Vegas.
} 
virtud de sus conocimientos" y con una compensación anual de 2,400 pesos cubanos (Secci 2014).

Una de las misiones de particular interés durante los años en los que dirige el departamento de botánica en la isla es la del 7 de marzo $[s i c]^{12}$ de 1921: el encargo, por parte del Ministro de Agricultura, de visitar junto a su esposo, director de la Estación, el jardín botánico de la Universidad de Harvard en la Central Soledad de Cienfuegos. En el documento, Mameli es comisionada también a visitar el jardín Cabada, en los alrededores de Cienfuegos, con el objetivo de traer a la Estación nuevas semillas, plantas y datos de interés (Secci 2014). Al regreso de la misión se publican dos artículos divulgativos. El primero, firmado por Mario, celebra los veinte años dedicados al jardín botánico por parte de Mr. Edwin F. Atkins y su ejemplar generosidad en el acto de donación en la Universidad de Harvard: "solo las personas civiles y de noble espíritu pueden cumplir actos semejantes y deberían ser ejemplo para muchos criollos ricos" (Calvino 1921: 542-546). El artículo está ilustrado con fotografías, en algunas de las cuales está presente Eva en la proximidad de la laguna que formaba parte del jardín botánico o junto a un árbol de caoba de una decena de años. El artículo prosigue en el número sucesivo de la revista (568-569) y continúa describiendo aquel agradable viaje. Eva aparece orgullosa en todas las fotos que la retratan: una en el palmar del jardín botánico, otra entre dos colosales pandanus con raíces aéreas, otra con la mirada dirigida al cielo con un elegante sombrero en medio de una palma de hojas glaucas, como describe Mario. En una de las fotos Eva está sentada en un quiosco rodeado de vegetación, muy parecido al del chalet contiguo al recinto de la Estación en Santiago de las Vegas. El quiosco de su casa (destruida por un ciclón en 1926, un año después de su regreso a Italia) aún existe hoy. De su residencia queda solo aquel merendero y unas pocas gradas suspendidas como recuerdo.

En el mismo número de la revista Eva publica el segundo artículo, que trata sobre la visita a Cienfuegos. El objetivo es un homenaje a la obra educativa de la esposa de Mr. Atkins, Mrs. Katherine, cuyo nombre la autora dice querer señalar por admiración y como ejemplo "para las señoras ricas". Eva describe cuidadosamente la organización de la escuela de costura para niñas y señoritas del lugar fundada por Kathe-

${ }^{12}$ La copia del documento en el Expediente Eva Mameli reporta “mayo". 
rine que, a través de un sistema de ahorro y de venta de los productos en el negocio de la Central, asegura una pequeña ganancia para las participantes. Mameli se une así al interés de Mario por la dedicación didáctica en el ambiente rural, y sugiere a las personas pudientes, igual que en el citado artículo de Calvino, nobles soluciones de compromiso social (Mameli 1921b: 564-565).

El año 1922 es el de las misiones fuera de Cuba. Es del 22 de junio (Secci 2014) la comunicación para Eva en la que un decreto presidencial le concede hasta cuatro meses de misión a Pavía, en cuyo Instituto Botánico de la Real Universidad podrá identificar las plantas que hasta ahora no han sido catalogadas por la falta de obras de consulta. En el mismo documento se le autoriza, sin remuneración adicional a la recibida como jefe del departamento de botánica de la Estación, a unirse a la visita que Mario Calvino realizará a Brasil, con el fin de que lo ayude a profundizar en los métodos de cultivo de la yuca y de otras plantas que sería oportuno cultivar en Cuba. El detallado testimonio científico de la misión esta vez parece ser escrito a cuatro manos. ${ }^{13}$ En el reportaje se enlistan fechas y etapas: el 2 de julio la pareja parte para Italia, en donde se detiene solo por ocho días durante los cuales — cuentan los protagonistas - asisten a una huelga ferroviaria y a la consiguiente reacción fascista y a un tiroteo en las calles de Génova. Los Calvino viven una serie de peripecias durante aquel viaje que les obliga a anticipar la partida a Brasil, logrando de todas maneras sacar algunas semillas y datos para su experimentación posterior en Cuba. Salen el 5 de agosto hacia Brasil, haciendo escala en Barcelona. En la siguiente escala, Dakar, no se les permite desembarcar a causa de una epidemia de cólera. Llegan a Río de Janeiro el 20 de agosto y visitan São Paulo a principios de septiembre. La estancia fue muy provechosa, según lo declarado por los mismos cónyuges en el artículo, y en el viaje de regreso, gracias a una escala en New York, logran visitar el jardín botánico del Bronx Park. Es del primero de noviembre de 1922 el documento que anuncia la reanudación laboral oficial de Eva Mameli en la Estación (Secci 2014).

El Expediente Eva Mameli reconstruye también otro momento importante de la vida cubana de Eva: el 15 de octubre de 1923 nace el primogénito Italo, en el chalet ubicado dentro de la misma Estación.

${ }^{13}$ Mameli y Calvino: 35-40. Un artículo sobre el cultivo del tabaco en Brasil, en el mismo número, es firmado sólo por Mario Calvino. 
Después de pocos días, la nueva mamá recibe la autorización firmada por el viceministro de agricultura G. Schwey en la que se le concede lo que ella misma había solicitado: una licencia pagada por "cuestiones personales". Algunos meses después, el 4 de febrero de 1924, el mismo viceministro le concede una licencia por enfermedad (Secci 2014). Son de Italo las palabras:

De Cuba no recuerdo nada, desgraciadamente, porque en 1925 ya estaba yo en Italia, en San Remo [...] De mi nacimiento al otro lado del océano no conservo más que un dato del registro civil difícil de transcribir, una maleta de memorias familiares, y el nombre de bautizo, inspirado en la pietas de los emigrantes hacia los propios lares y que en la patria en cambio resuena bronceo y carducciano (Calvino 1956).

La permanencia en Cuba de los Calvino concluye en 1925, cuando Mario es llamado a Italia para fundar y dirigir la Estación Experimental de San Remo, abriendo una nueva etapa en la sociedad Mameli-Calvino. El regreso a Italia abrirá también otro gran capítulo en la vida de los dos científicos: se deberá a ambos la intensificación de la floricultura en las provincias de San Remo e Imperia y los injertos innovadores de plantas y flores, sin descuidar nunca las iniciativas de educación y divulgación.

Pero ciertos vínculos, ratificados por la memoria, no se pierden más allá de las fronteras; como aquellos que tienen que ver con la tierra. He aquí un extracto de la carta que el 15 de diciembre de 1951 Eva Mameli envía desde San Remo a Antonio Portuondo (Secci 2014), director de la Estación Experimental Agronómica de Santiago de las Vegas, en respuesta a un mensaje de condolencias. La muerte de Mario no disuelve la sociedad, más bien la refuerza: "Muy distinguido Ing. Portuondo, quiero expresarle mi agradecimiento por su carta de pésame. La profunda pena me ha impedido de participar a todos los amigos de Cuba la triste noticia, pero puede vd. tener la seguridad que, como mi esposo siempre recordaba a Cuba con afecto sincero y grato, en mí también su país, y en modo espacial la Estación Agronómica, han dejado una estela de gratos recuerdos. Tengo la suerte de poder continuar el trabajo de mi esposo en floricultura y este es un alivio a mi grande dolor" (Secci 2014).

Una estela de bellos recuerdos que traza el mapa verde de dos viajeros científicos, padres de uno de los escritores italianos más apreciados en el mundo, hijo también del Caribe. 


\section{BibLIOGRAFÍA}

Adami, Stefano. "Lombra del padre. Il caso Calvino", en California Italian Studies Journal, University of California, vol. 1, núm. 2, 2010.

Aicardi, Domenico. Mario Calvino (Inédito).

Calvino, Italo. "Lettera al prof. Angelo Tamborra", en Lettere 1940-1985. Milán: Mondadori, 2000: 1379.

Calvino, Italo. "Questionario 1956”, en Il Caffe, IV, núm. 1, enero 1956.

Calvino, Italo. Eremita a Parigi. Pagine autobiografiche. Milán: Mondadori, 1994.

Calvino, Mario. "Discurso del Prof. Mario Calvino en la inauguración de la 'Escuela de Agricultura de Yucatán', el 5 de diciembre de 1915". $<$ http://www.poresto.net/ver_nota.php?zona=yucatan\&idSeccion=1\&i dTitulo $=64149>$.

Calvino, Mario. "La Estación Botánica del Central 'Soledad' de Cienfuegos", en Revista de Agricultura, Comercio y Trabajo, La Habana, vol. 4, núm. 4, año IV (abril 1921): 542-546.

Calvino, Mario. Nota en La Agricoltura Ligure, 31 de diciembre de 1908.

Díaz Marreno, Concepción. "Aportes italianos a la agricultura de Cuba y otros países vecinos durante la primera mitad del siglo xx", en Emigrazione e presenza italiana in Cuba. Roccarainolla, Circolo Culturale B. G. Duns Scoto, vol. III (2004): 89-116.

Maceri, Gerson. Mario Calvino, biografia di un progressista utopico. San Remo: Quaderni Sanremesi, 2012.

Mameli Calvino, Giuliana Eva. Mario Calvino 1875-1951. Roma: Istituto Poligrafico dello Stato, 1952.

Mameli, Eva. "Las mujeres en los Institutos Científicos de Pavía, Italia", en Revista de Agricultura, Comercio y Trabajo, La Habana, núm. 6, año IV (junio 1921a).

Mameli, Eva. "La obra educadora de Mrs Katherine Atkins", en Revista de Agricultura, Comercio y Trabajo, La Habana, vol. 4, núm. 5, año IV (mayo 1921b): 564-565.

Mameli, Eva y Mario Calvino. "Informe del viaje a Brasil", en Revista de Agricultura, Comercio y Trabajo, La Habana, vol. 5, núm. 6, año VII, 1923.

Mameli, Eva y Gino Pollacci. Note critiche intorno a recenti ricerche sulla fotosintesi clorofilliana, Actas del R. Instituto botánico de la Universidad de Pavía, Rebeschini di Turati e C., Milan [posteriormente al 1908].

Mameli, Eva y Gino Pollacci. Sull'assimilazione diretta dell'azoto atmosferico libero nei vegetali, Instituto botánico de la R. Universidad de Pavía y 
Laboratorio Criptogámico italiano, Actas del Instituto botánico de la R. Universidad de Pavía, S. 2., V. 14, Rebeschini e C., Milan, 1911.

Marchi, LoRetTa. "I giardini di Eva”, en Federica Frediani, Ricciarda Ricorda, Luisa Rossi, eds., Spazi, segni, parole. Percorsi di viaggiatrici italiane. Milan: Franco Angeli, 2012.

Nicolas, Jean Henri. A Rose Odyssey. Reminiscence of Many Trips to European Rose Centers. Nueva York: Doubleday, Doran \& Co. Inc., 1937.

Secci, M. Cristina. "Eva Mameli: le piante, il mio dovere, la mia passione", en Altre Modernità, núm. 10-11, Milán, 2013.

Secci, M. Cristina. Introducción, en E. Mameli Calvino y M. Calvino. 250 preguntas y respuestas sobre jardinería. México: DGE Equilibrista, 2013.

Secci, M. Cristina. Eva Mameli Calvino: de Cerdeña a América Latina con microscopio y familia. Registro digitale di 1700 immagini degli archivi di Santiago de las Vegas. Ciudad de México: Consejo Nacional de Ciencia y Tecnología / Fondazione Banco di Sardegna / Istituto Italiano di Cultura, 2014.

Valle, Nicola. "Eva Mameli”, en L'Unione Sarda, Cagliari, 23 de febrero de 1969. 